\title{
Fast collisional electron heating and relaxation in thin foils driven by a circularly polarized ultraintense short-pulse laser
}

\author{
Andréas Sundström ${ }^{\circledR 1} \uparrow$, Laurent Gremillet ${ }^{\circledR 2}$, Evangelos Siminos ${ }^{\circledR 3}$ \\ and István Pusztai ${ }^{\circledR 1}$ \\ ${ }^{1}$ Department of Physics, Chalmers University of Technology, 41296 Gothenburg, Sweden \\ ${ }^{2}$ CEA, DAM, DIF, F-91297 Arpajon, France \\ ${ }^{3}$ Department of Physics, University of Gothenburg, 41296 Gothenburg, Sweden
}

(Received 21 November 2019; revised 17 March 2020; accepted 18 March 2020)

The creation of well-thermalized, hot and dense plasmas is attractive for warm dense matter studies. We investigate collisionally induced energy absorption of an ultraintense and ultrashort laser pulse in a solid copper target using particle-in-cell simulations. We find that, upon irradiation by a $2 \times 10^{20} \mathrm{~W} \mathrm{~cm}^{-2}$ intensity, $60 \mathrm{fs}$ duration, circularly polarized laser pulse, the electrons in the collisional simulation rapidly reach a well-thermalized distribution with $\sim 3.5 \mathrm{keV}$ temperature, while in the collisionless simulation the absorption is several orders of magnitude weaker. Circular polarization inhibits the generation of suprathermal electrons, while ensuring efficient bulk heating through inverse bremsstrahlung, a mechanism usually overlooked at relativistic laser intensity. An additional simulation, taking account of both collisional and field ionization, yields similar results: the bulk electrons are heated to $\sim 2.5 \mathrm{keV}$, but with a somewhat lower degree of thermalization than in the pre-set, fixed-ionization case. The collisional absorption mechanism is found to be robust against variations in the laser parameters. At fixed laser pulse energy, increasing the pulse duration rather than the intensity leads to a higher electron temperature.

PACS: plasma simulation, plasma heating, plasma dynamics

\section{Introduction}

The creation of warm dense matter (WDM) or hot dense matter (HDM) in a laboratory setting is of high interest for a broad field of research disciplines such as laboratory astrophysics (Remington 2005; Bailey et al. 2007; Fujioka et al. 2009), studies of planetary interiors (Ross 1981; Knudson, Desjarlais \& Dolan 2008), inertial confinement fusion (Drake 2018; Le Pape et al. 2018), understanding the equations of state under such extreme conditions (Renaudin et al. 2003; Nettelmann et al.

$\dagger$ Email address for correspondence: andsunds@chalmers.se 
2008) and experimental verification of high energy density (HED) atomic physics models (Hoarty et al. 2013b; Faussurier \& Blancard 2019). However, in order to benchmark atomic physics models against spectroscopic data, these must be obtained under well-controlled conditions. Since most such models assume Maxwellian plasma populations, this means that, when diagnosed, the heated samples should be as close to thermal equilibrium as possible.

The generation of WDM/HDM at uniform near-solid density requires that the sample be heated rapidly, i.e. before any significant hydrodynamic expansion. Such isochoric heating can be achieved using ultrahigh-intensity, short-pulse lasers, as has been done at various high-power systems (Evans et al. 2005; Gregori et al. 2005; Martinolli et al. 2006; Chen et al. 2007; Nilson et al. 2009; Pérez et al. 2010; Brown et al. 2011; Hoarty et al. 2013a). These experiments were conducted using laser pulses with $0.3-10$ ps duration and energies in the range of 10-500 J, but there is a need for a wider access at lower-energy table-top facilities, typically delivering joule-level, femtosecond laser pulses. Promising results in this direction have recently been obtained by Purvis et al. (2013) and Bargsten et al. (2017) making use of nano-wire arrays to strongly enhance the laser-to-plasma coupling efficiency, thus creating keV temperature, sub-solid density plasmas. Yet, such structured targets are non-trivial to manufacture and are extremely sensitive to parasitic laser prepulses, which can destroy the nano-structures before the main pulse arrives.

Most laser-based isochoric heating experiments conducted so far have exploited the fast electrons driven by a linearly polarized laser pulse (Nilson et al. 2010; Santos et al. 2017; Sawada et al. 2019). Their energy dissipation through the plasma bulk enables heating to high temperatures $(0.1-1 \mathrm{keV})$ at solid-range plasma densities, but usually at the expense of poor spatial uniformity (Dervieux et al. 2015) and relatively slow thermalization. Plasma heating in this case is caused by the interaction of the fast electrons with the bulk plasma via a combination of direct collisions (Robinson et al. 2014), ohmic dissipation of the colder return current (Lovelace \& Sudan 1971; Guillory \& Benford 1972; Bell \& Kingham 2003; Robinson et al. 2014) or plasma waves driven by the fast electrons (Sherlock et al. 2014). Some experiments have been done with laser-accelerated ions to heat a secondary target (Patel et al. 2003; Dyer et al. 2008; Mančić et al. 2010). Yet, while this heating method can provide better spatial uniformity, it leads to much lower $(\sim 10 \mathrm{eV})$ temperatures.

At normal laser incidence and linear polarization (LP), and for sharp-gradient, highly overdense plasmas, the most commonly invoked mechanisms of laser energy conversion into fast electrons are $j \times B$ heating (Kruer \& Estabrook 1985) and vacuum heating (Bauer \& Mulser 2007; May et al. 2011). Both mechanisms hinge on the temporal modulation of the laser ponderomotive force around the target surface, and thus lead to periodic injection of $\mathrm{MeV}$ range electron bunches into the target at twice the laser frequency. Such suprathermal electrons thermalize relatively slowly $(\sim \mathrm{ps})$, which may hinder those applications that require a closely Maxwellian dense plasma. In a work by Kemp \& Divol (2016), it is shown that the fast electron bunches induce surface waves that can scatter the energized bulk electrons, thereby improving absorption. They also show the necessity of collisions to first heat up the target surface to $\mathrm{keV}$ temperatures, required for vacuum heating to commence.

By contrast, using laser pulses with circular polarization $(\mathrm{CP})$, for which the ponderomotive force does not show high-frequency oscillations, the $j \times B$ and vacuum heating mechanisms are essentially suppressed in overdense targets, and so is the fast electron bunch production (and the surface waves induced by them). Still, some fast electrons can be produced with $\mathrm{CP}$ if the variation time scale of the laser envelope is not large compared to the laser cycle (Siminos et al. 2012, 2017). 
In this paper, we study the effects of collisions on the energy absorption capability of the electrons in a thin, solid foil of a high-atomic-number element. Due to the high atomic number, it is not clear a priori what degree of ionization $\left(Z^{*}\right)$ the ions have throughout the process and what influence the ionization history has on heating. While a high $Z^{*}$ is desirable for the collisional heating process, the initially cold target will not be highly ionized in the beginning. Therefore, we have studied both different degrees of fixed ionization as well as the self-consistent ionization process including both field and impact ionization.

We demonstrate that the energy absorption of an intense short laser pulse in a high- $Z^{*}$ solid-density target is mainly due to inverse bremsstrahlung electron heating within the plasma skin layer, and that this scenario holds in a broad range of experimentally relevant parameters. The front-layer electrons are collisionally scattered into the target body where they heat the plasma bulk to $\mathrm{keV}$ level temperatures, enough to reach Gbar range pressures, which is well in the regime of HDM. The scattered electrons have sufficiently low energies that they primarily heat the bulk via direct collisional thermalization. Since this mechanism relies on the scattering of the electrons accelerated by the laser field against the heavy ions, it is operative regardless the polarization. Inside the plasma, where the laser field is negligible, collisions cause fast relaxation of the electron distribution to a Maxwellian.

\section{Simulation design}

We have performed one- and two-dimensional (1-D and 2-D respectively) particle-incell (PIC) simulations of laser-solid interactions with and without collisions enabled. We have used the Smilei PIC code (Derouillat et al. 2018), which has a relativistic binary collision module (Pérez et al. 2012) based on the collisional algorithm by Nanbu (1997) and Nanbu \& Yonemura (1998). In the case of a collisional plasma, we have considered either a fixed degree of ionization or self-consistent modelling of the ionization process - through field ionization and collisional impact ionization.

We ran 1-D simulations in a box of size $20 \mu \mathrm{m}$ with a resolution of $\Delta x=0.39 \mathrm{~nm}$ (51200 cells). We considered both LP and CP laser pulses with wavelength $\lambda=800 \mathrm{~nm}$, dimensionless amplitude ${ }^{1} a_{0}=10$ (intensity $I=\frac{1}{2} c \epsilon_{0}\left(m_{\mathrm{e}} c \omega a_{0} / e\right)^{2} \approx$ $2 \times 10^{20} \mathrm{~W} \mathrm{~cm}^{-2}$, where $\epsilon_{0}$ is the vacuum permittivity, $m_{\mathrm{e}}$ the electron mass, $e$ elementary charge and $\omega$ the laser frequency) and a Gaussian temporal profile with $t_{\mathrm{FWHM}}=60 \mathrm{fs}$ full-width-at-half-maximum (FWHM) duration in the intensity. The plasma is $2.5 \mu \mathrm{m}$ thick, starting at $x=7.5 \mu \mathrm{m}$ with a linear density ramp over a distance of $20 \mathrm{~nm}$. The plasma consists of electrons and copper ions at solid density, $n_{\mathrm{Cu}, 0}=48.4 n_{\mathrm{c}} \approx 8.4 \times 10^{22} \mathrm{~cm}^{-3}$, with 400 macro-particles per cell for each species. Here, $n_{\mathrm{c}}=\epsilon_{0} m_{\mathrm{e}} \omega^{2} / e^{2}$ is the critical density associated with the laser frequency $\omega$. The particles are initialized from Maxwell-Jüttner distributions (in three momentum dimensions) with temperatures $T_{\mathrm{e}, 0}=1 \mathrm{eV}$ for the electrons and $T_{\mathrm{i}, 0}=0.1 \mathrm{eV}$ for the ions.

In order to assess the influence of the plasma collisionality alone, we have first carried out simulations with fixed ionization degrees $Z^{*}=11,19,24$ and 27. Then, to ascertain the physical accuracy of these results, we have performed simulations describing both collisional and field ionization. The collisionless skin depth $l_{\mathrm{s}}=c /\left[\omega\left(n_{\mathrm{e}} / n_{\mathrm{c}}\right)^{1 / 2}\right]$ is resolved, even for the highest ionization where

\footnotetext{
${ }^{1}$ Note that the amplitude is normalized such that the intensity stays the same regardless of ellipticity, i.e. the field amplitude, at the same $a_{0}$ with circular polarization is $E_{\mathrm{CP}}=E_{\mathrm{LP}} / \sqrt{2}$ compared to that of $\mathrm{LP}$, $E_{\mathrm{LP}}$.
} 


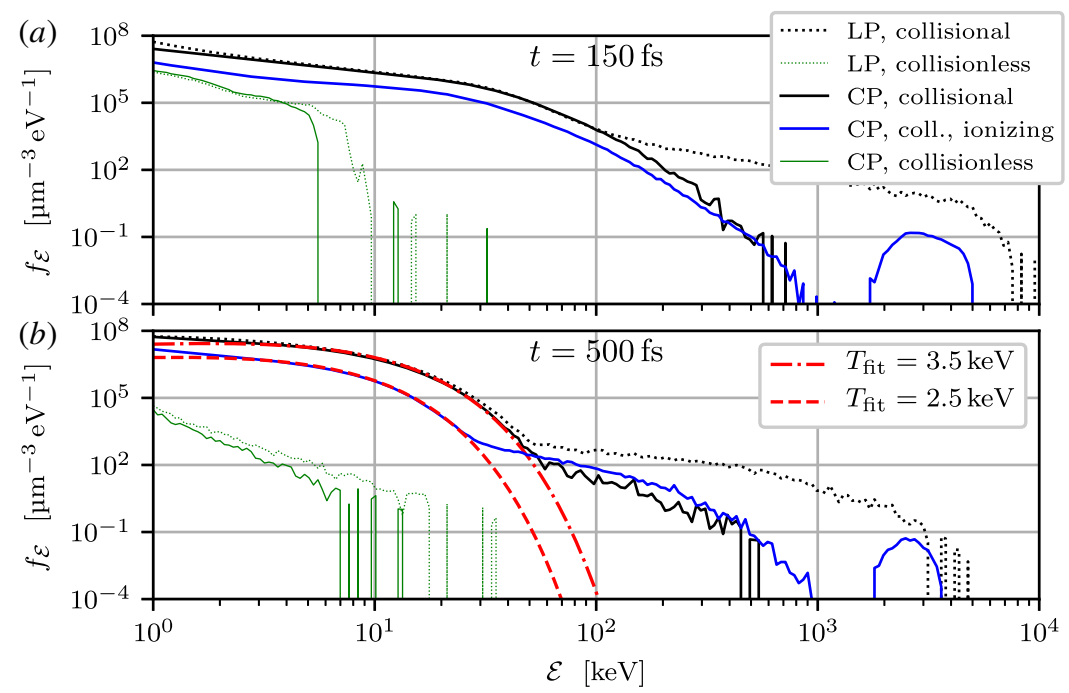

FIGURE 1. Electron energy spectra $f_{\mathcal{E}}$ at times $t=150$ fs $(a)$ and $t=500$ fs $(b)$, for LP (dotted lines) and CP (solid lines), with (black lines) and without collisions (thin, green lines); also showing CP with self-consistent field and collisional ionization (blue, solid line). Two Maxwell-Jüttner distributions are fitted to the bulk of the CP self-consistent and fixed-ionization electron spectra in panel $(b)$ (red dashed and dash-dotted lines respectively).

$l_{\mathrm{s}}^{\left(Z^{*}=27\right)} \approx 3.5 \mathrm{~nm}$. The values of $Z^{*}=11,19$ and 27 correspond to full depletion of different electronic shells, $Z^{*}=27$ being the reference ionization used in other scans. An additional data point, $Z^{*}=24$, was chosen as an arbitrary value between 19 and 27. When modelling the ionization process self-consistently, the ions were initialized with $Z_{0}^{*}=5$, in accordance with the widely used Thomas-Fermi model (More 1983). Both field-tunnelling and electron-ion impact ionization were enabled. The self-consistent ionization simulation was only performed with $\mathrm{CP}$.

We also performed one collisional 2-D simulation to check that our results are robust to multidimensional effects. This simulation uses the same $\mathrm{CP}$ laser and target parameters as our 1-D base case. In order to limit the computational cost at the increased dimensionality, it was performed at a reduced resolution of 640 cells per micron in both directions $(\Delta x=\Delta y=1.56 \mathrm{~nm})$, and a simulation box size of $10 \mu \mathrm{m}$ longitudinally and $1.6 \mu \mathrm{m}(2 \lambda)$ transversely. Furthermore, the number of particles per cell per species was reduced to 50. A test of these resolution parameters in one dimension showed excellent agreement in electron kinetic energy spectrum of the main body of the electrons with the corresponding high-resolution, collisional 1-D simulation; however, the lower particle count led to a poorer statistics in the high-energy tail of the electron spectrum.

\section{Results and discussion}

Figure 1 compares the electron energy spectra as obtained at $\mathrm{CP}$ and $\mathrm{LP}\left(Z^{*}=\right.$ 27), with or without collisions enabled and at $\mathrm{CP}$ with self-consistent ionization. The spectra are recorded at two successive times $(t=150 \mathrm{fs}$ and $t=500 \mathrm{fs})-$ for reference, the peak laser intensity hits the target at $t \approx 110 \mathrm{fs}$ and the pulse FWHM duration is 
60 fs. For both types of polarization, much higher electron energies are achieved when allowing for collisions. The collisionally enhanced absorption results in a bulk electron temperature of $T_{\mathrm{e}} \approx 3.5 \mathrm{keV}$ at $t=500$ fs for both LP and CP, determined by fitting Maxwell-Jüttner distributions to the bulk spectra (i.e. ignoring the tails). Meanwhile, the collisionless simulations only reach an electron temperature of $\sim 10-100 \mathrm{eV}$; these electrons are, however, far from being thermalized and only their energetic tails are visible in the figure. The fact that both CP and LP reach very similar bulk electron temperatures when collisions are enabled indicates that the laser absorption mechanism is the same in both cases.

By comparing the electron spectra with the fitted Maxwellians, we conclude that the electrons have reached a degree of thermalization wherein less than $0.1 \%$ of the kinetic energy is in the high-energy tail for CP, and $\sim 1 \%$ for LP. The time frame for this thermalization is consistent with a rough estimate for electron-electron collisional energy transfer rates. At $t=500 \mathrm{fs}$, the deviation from Maxwellian starts at an electron energy $\mathcal{E} \simeq 50 \mathrm{keV}$. This is consistent with the $\sim 300$ fs collisional energy relaxation time of a $50 \mathrm{keV}$ electron through a $3.5 \mathrm{keV}$ plasma at $2.3 \times 10^{24} \mathrm{~cm}^{-3}$ electron density (Huba 2016).

Note that these results compare LP and CP for the same on-target intensity, while in an experiment, the circularly polarized pulse would in practice be at a somewhat lower intensity due to losses in the conversion from LP to CP (through a quarter-wave plate). Another practical concern may be elliptical polarization, due to imperfections in the $\mathrm{CP}$ conversion. While the exact dependence of the fast electron generation on the ellipticity of the polarization is non-trivial, the bulk collisional absorption itself is not expected to be affected by the ellipticity, as demonstrated by the same bulk temperatures reached in the two extreme cases of CP and LP.

The electron temperatures we quote are technically calculated before the electrons have fully thermalized with the ions, which occurs over $\sim$ ps time scales. However, due to the high degree of ionization, the electron-ion equilibrium temperature is $T_{\mathrm{eq}} \approx n_{\mathrm{e}} /\left(n_{\mathrm{e}}+n_{\mathrm{i}}\right) T_{\mathrm{e}} \approx 0.96 T_{\mathrm{e}}$. Thus, energy transfer from the electrons to the ions is insignificant.

When activating self-consistent (both field and collisional) ionization, the bulk electron temperature is slightly reduced $(\sim 2.5 \mathrm{keV})$ compared to the fixed-ionization case. The front plasma is rapidly ionized, mostly through field ionization, so that collisional absorption quickly reaches an efficiency similar to that obtained with fixed $Z^{*}=27$ (see figure $2 b$ showing that the average ionization $\left\langle Z^{*}\right\rangle \simeq 24$ at the plasma front already at $t=100 \mathrm{fs}$ ). The lower $T_{\mathrm{e}}$ is mostly due to the energy spent on ionization - the average ionization energy from $Z^{*}=5$ to 27 is $0.9 \mathrm{keV}$.

Moreover, figure 1(b) shows that, for both CP and LP, collisions cause efficient bulk electron thermalization as early as $t=500 \mathrm{fs}$. High-energy tails are found to emerge above $\sim 50 \mathrm{keV}$ for the fixed ionization and $\sim 30 \mathrm{keV}$ for the self-consistent ionization. Note the large range of the logarithmic $f_{\mathcal{E}}$ scale, meaning that the tails are three to five orders of magnitude lower than the bulk spectra. The non-thermal tail is heavier in LP than in $\mathrm{CP}$, due to the operative $j \times B$ and vacuum heating.

Also, the simulation with self-consistent ionization displays a larger tail, compared to the bulk spectrum, than its counterpart with fixed $Z^{*}$. The larger tail as well as an electron population at $\sim 3 \mathrm{MeV}$ can be explained by field-ionization events in the charge-separation layer, which is exposed to stronger laser fields. As the target front electrons are being pushed back by the ponderomotive force, the ions remaining in the charge-separation layer experience the less shielded laser field which quickly ionizes them further. Since these newly freed electrons are injected into regions of stronger 
(a)

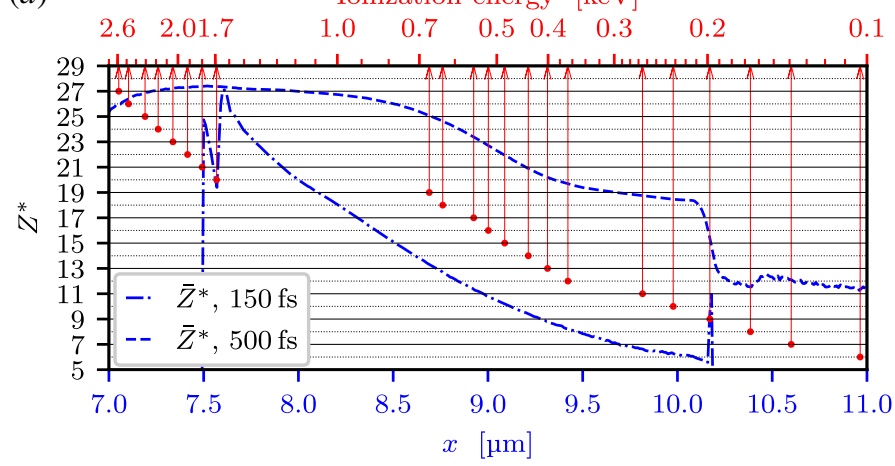

(b)

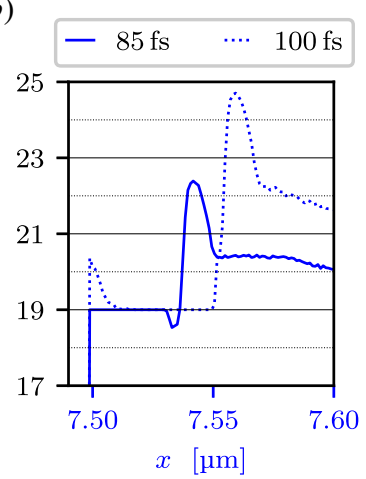

FIgURE 2. Average ionization level profiles $\bar{Z}^{*}$ in the self-consistent field and collisional ionization simulation (blue lines, bottom axis) at times $t=150 \mathrm{fs}$ (dash-dotted line) and $t=500 \mathrm{fs}$ (dashed line), and ionization level as a function of ionization energies of copper (red dots and arrows, top axis) - ionization data obtained from the Atomic Spectra Database of the National Institute of Standards and Technology (Kramida et al. 2018). Panel $(b)$ shows the average ionization level profiles $\bar{Z}^{*}$ near the target front at $t=85 \mathrm{fs}$ (solid line) and $t=100 \mathrm{fs}$ (dotted line).

laser fields, they are energized similarly to vacuum heating in LP, thus resulting in a larger population of non-thermal electrons, which, as in LP, thermalize relatively slowly. Furthermore, the average ionization level is lower inside the target with selfconsistent ionization, as seen in figure 2, thus reducing the efficacy of collisional thermalization. Both these effects act to give a larger high-energy tail.

The ionization level profiles $\left(\bar{Z}^{*}\right)$ of the self-consistent ionization simulation shown in figure 2(a) represent the local ionization levels averaged over all macro-particles in each spatial cell. Comparing the $t=150 \mathrm{fs}$ (dashed-dotted line) and the $t=500 \mathrm{fs}$ (dashed line) average ionization curves, we see that the target front quickly reaches a high ionization degree while the bulk is ionized more gradually. Since there are no strong electric fields inside the plasma, the ionization of the bulk must be driven by impact ionization. The $\bar{Z}^{*}$ curve at $t=500 \mathrm{fs}$ (dashed line) in figure $2(a)$ displays plateaus at $\bar{Z}^{*}=27,19$ and to some extent 11 . These plateaus result from the large jumps in ionization energies between the successive outermost electron shells (e.g. between $\bar{Z}^{*}=11$ and 12 , or $\bar{Z}^{*}=19$ and 20 ; see figure $2 a$ ). The ionization energy to reach $\bar{Z}^{*}=28$ is $\sim 11 \mathrm{keV}$

Figure $2(b)$ shows $\bar{Z}^{*}$ at the target front surface $(x=7.5 \mu \mathrm{m})$. At time $t=85 \mathrm{fs}$ (solid line), the ionization level has saturated at $\bar{Z}^{*}=19$ due to the jump in ionization energy after $Z^{*}=19$. Later, at $t=100 \mathrm{fs}$ (dotted line), the laser field has become strong enough to sustain field ionization beyond $Z^{*}=19$, yielding the peak in $\bar{Z}^{*}$ near $x=7.5 \mu \mathrm{m}$. Apart from the laser field, the electrostatic field $\left(E_{x}\right)$ induced by the laser ponderomotive force at the target front causes additional ionization. This results in the $\bar{Z}^{*}$ peak seen around $x=7.55 \mu \mathrm{m}$, which moves into the plasma as the chargeseparation layer is pushed forward by the laser ponderomotive force.

The difference between the simulations is made clearer when studying the electron phase spaces shown in figure $3 .^{2}$ The figure displays time sequences of the collisional

\footnotetext{
${ }^{2}$ The normalization $f / f_{\max }$ of the distribution functions in figures 3 and 5 are with respect to the initial maximum value of the distribution function in their respective planes of phase space $f_{\max }$. The colour values of the plotted distributions can therefore be directly compared.
} 

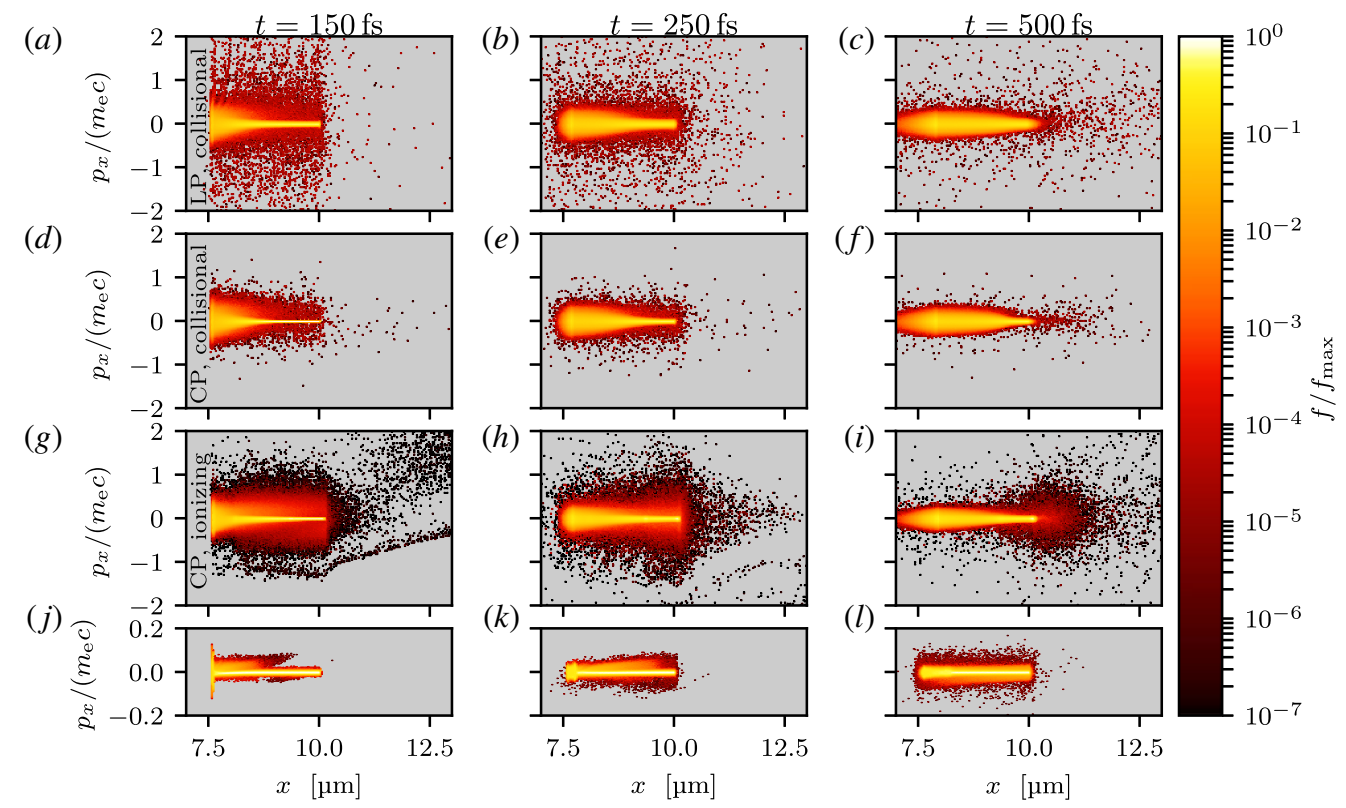

FIGURE 3. Electron phase-space distributions for collisional LP with fixed ionization $(a-c)$; collisional CP with fixed ionization $(d-f)$ and self-consistent field and collisional ionization $(g-i)$ as well as for collisionless CP $(j-l)$, at times $t=150 \mathrm{fs}(a, d, g, j), 250 \mathrm{fs}$ $(b, e, h, k)$ and $500 \mathrm{fs}(c, f, i, l)$. Note the different momentum scale for the collisionless CP.

distributions with LP in the top row and CP in the second row; the third row shows the self-consistent ionization CP simulation and the bottom row shows the collisionless CP distribution. In the LP simulation, high-energy electron bunches are produced at twice the laser frequency, as seen in the $t=150$ fs panel (top row), while CP with fixed ionization (second row) produces a more even distribution of hot electrons since $j \times B$ and vacuum heating mechanisms are inhibited. At $t=500 \mathrm{fs}$, most of the fast electrons have thermalized in the fixed-ionization case, while there remains a significant population of high-energy electrons 'swarming' around the back of the target with self-consistent ionization.

With self-consistent ionization, two populations of relatively high-energy electrons are created during the rising phase of the laser pulse. These populations originate from two successive field-ionization phases. The first one occurs early in the interaction, when the ionization of the surface plasma momentarily saturates at $\bar{Z}^{*}=19$. In the $t=150$ fs panel of figure 3 , this population accounts for the broad momentum distribution in the target bulk, and also for the beam (with momenta $p_{x} /\left(m_{\mathrm{e}} c\right) \sim-1$ ) being reflected in the vacuum $(x>10 \mu \mathrm{m})$ and refluxing into the target. The second phase starts at $t \simeq 90 \mathrm{fs}$, when the laser pulse gets intense enough to ionize the surface plasma beyond $Z^{*}=19$ (compare the $85 \mathrm{fs}$ and $100 \mathrm{fs}$ curves in figure $2 b$ ). This yields fast electrons (visible in the upper right corner of the $t=150$ fs phase space) more energetic than those generated earlier, which correspond to the bump around $\sim 3 \mathrm{MeV}$ in the energy spectra of figure 1. A similar field-ionization injection of fast electrons from the surface ions was seen by Kawahito \& Kishimoto (2017) in a carbon plasma, although they used LP which also caused bunching of the electrons at twice the laser frequency. 

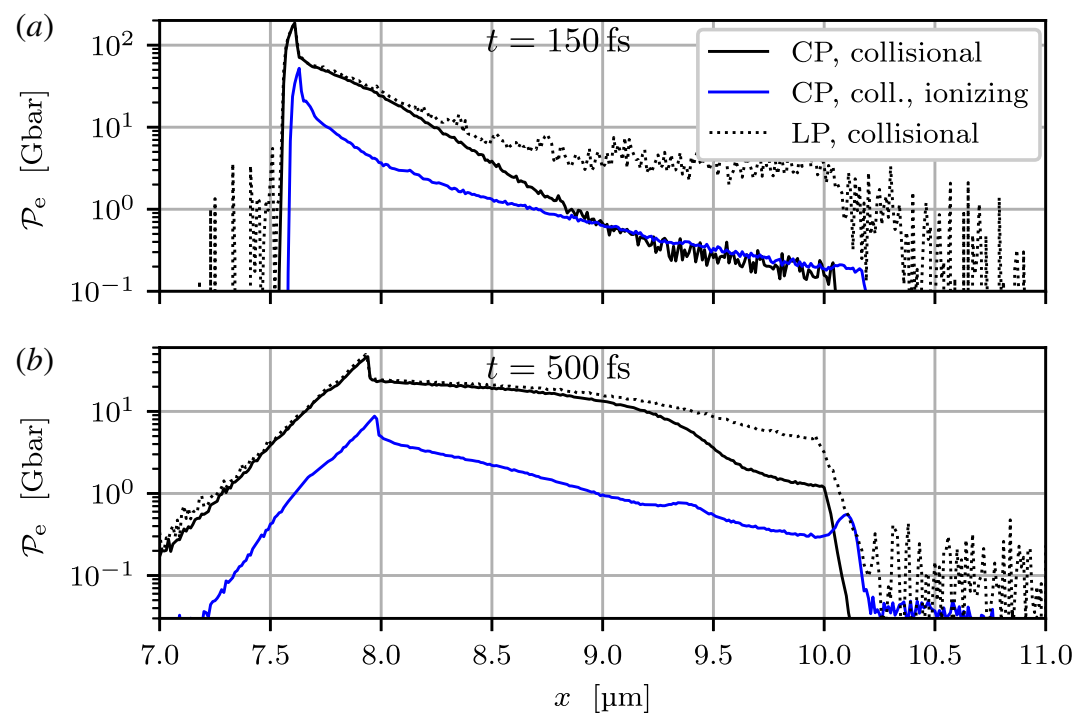

FIGURE 4. Electron kinetic energy density $\mathcal{P}_{\mathrm{e}}$ for collisional LP (dotted line) and CP (black solid line) and CP with self-consistent field and collisional ionization (blue solid line), at times $t=150 \mathrm{fs}(a)$ and $t=500 \mathrm{fs}(b)$. The peak laser intensity hits the target at $t \approx 110 \mathrm{fs}$.

We now turn our attention to the energy density achieved in these scenarios. Since the heating process is fast compared to hydrodynamical time scales, the plasma bulk has not had time to expand, and hence the bulk electrons and ions remain at solid-range density. At the same time, the electrons reach $\mathrm{keV}$ temperatures, resulting in high energy densities of the order of $\sim 10 \mathrm{Gbar}=10^{9} \mathrm{~J} \mathrm{~cm}^{-3}$. In figure 4 , the electron kinetic energy density $\mathcal{P}_{\mathrm{e}}$ is displayed throughout the target at times $t=150 \mathrm{fs}$ (top panel) and $t=500 \mathrm{fs}$ (bottom panel). At the earlier time, the kinetic energy density is concentrated to the front of the target, while at the later stage the energy has spread out throughout most of the plasma. The energy density in the fixed-ionization simulations reaches approximately $\sim 10$ Gbar and is mostly homogeneous in the region $x=8-9 \mu \mathrm{m}$.

The high-energy electrons created with LP facilitate a better spatial homogenization of the energy density than with $\mathrm{CP}$. Their slow thermalization results in a more spatially homogeneous target heating, since they can recirculate several times through the plasma. In a potential application, one should therefore make a compromise between good thermalization and homogenization. Another parameter that can be used to control homogenization is target thickness; decreasing it helps for a faster homogenization of the plasma heating. However, a thinner target will also explode faster hydrodynamically, which would give a HED application a shorter time frame to operate in.

Meanwhile, the self-consistent simulation gives an exponentially decreasing energy density profile throughout the target at $t=500 \mathrm{fs}$, indicating that thermalization is taking longer. The lower temperature and electron density reached with self-consistent ionization result in approximately an order of magnitude lower energy density compared to the fixed-ionization results. However, there is still a significant region with $\mathcal{P}_{\mathrm{e}}>1$ Gbar in the self-consistent ionization simulation at $t=500 \mathrm{fs}$. In this 
case, the energy density does not homogenize as efficiently, partly due to a decreased ability of the target to thermalize fast electrons (stemming from lower $\bar{Z}^{*}$ ), and partly due to the inhomogeneity of the ionization profile which affects the bulk electron density profile.

As a consequence of the strong gradients in $\mathcal{P}_{\mathrm{e}}$ around the target front side, a shock wave is launched. The shock wave presents itself as a sharp jump in electron pressure, most clearly seen close to $x=8.0 \mu \mathrm{m}$ in the $t=500 \mathrm{fs}$ panel in figure 4 . The details of shock formation are sensitive to the laser and target parameters, and are more clearly seen from the ion phase space, as will be addressed by a paper in preparation (Sundström et al. 2020). However, no ion reflection occurs at the shock front, which means that the shock is hydrodynamic like in its nature.

At the high ionization levels discussed in this paper, such high temperatures and densities may result in significant energy losses due to bremsstrahlung. The

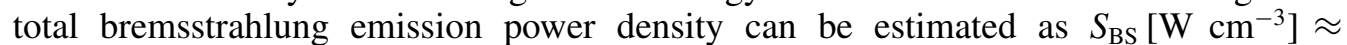
$1.69 \times 10^{32} \times Z^{* 3}\left(n_{\mathrm{i}}\left[\mathrm{cm}^{-3}\right]\right)^{2}\left(T_{\mathrm{e}}[\mathrm{eV}]\right)^{1 / 2}$ (Huba 2016). By comparing the power density to the thermal energy density $\sim \frac{3}{2} n_{\mathrm{e}} T_{\mathrm{e}}$, we arrive at a radiative time scale of the order of several ps for $\mathrm{keV}$ range temperatures at a density of $n_{\mathrm{i}}=8.4 \times 10^{22} \mathrm{~cm}^{-3}$. Hence, the radiative losses from bremsstrahlung will mostly be of concern at time scales longer than those studied in this paper. However, bremsstrahlung losses cannot be completely disregarded in a WDM/HDM experiments, where current spectroscopic temporal resolution is constrained to $\sim$ ps time scales.

Besides bremsstrahlung, line emission from relaxation of excited states may be of concern. However, at electron energies above $\gtrsim 10 \mathrm{eV}$, electron energy losses from excitations become subdominant compared to ionization (Joshipura, Vaishnav \& Limbachiya 2006). Since energy loss from collisional ionization events is accounted for in the self-consistent ionization simulation, the temperature of $T_{\mathrm{e}} \approx 2.5 \mathrm{keV}$ is likely not affected much by losses through line emissions.

\subsection{Illustration of the collisional absorption mechanism}

To illustrate the mechanism by which collisions enhance absorption, we have performed a simplified set of simulations. These are designed to generate a quasi-steady state: the laser intensity is constant after a linear ramp-up over 10 laser cycles; the ions are stationary; the plasma is $2.5 \mu \mathrm{m}$ long and it terminates at a thermal boundary, meaning that particles which exit the boundary are reflected with momenta chosen randomly from a Maxwellian distribution at $T_{\mathrm{e}, 0}=10 \mathrm{eV}$ for the electrons - the same at the initial temperatures. The other simulation parameters are: $\mathrm{CP}$ at $a_{0}=10, Z^{*}=27$ with and without collisions; resolution and other numerical parameters are as stated in $\S 2$. The long ramp-up time has been chosen to reduce electron energization due to the laser amplitude envelope modulation (Siminos et al. 2012). Note that due to the steady state nature of this simplified simulation setting, it is hard to draw any quantitative conclusions that can be transferred to the time-varying situation.

We will now take a look at the interaction between the electrons and the laser electric field. The density of power $S$ exerted on an electron population can be expressed as

$$
S(x, t)=-\mathrm{e} \int \mathrm{d}^{3} v \boldsymbol{E}_{\perp} \cdot \boldsymbol{v} f_{\mathrm{e}}(\boldsymbol{v})=-\mathrm{e} n_{\mathrm{e}} \boldsymbol{E}_{\perp} \cdot \boldsymbol{V}_{\perp},
$$

where $\boldsymbol{E}_{\perp}=\boldsymbol{E}_{\perp}(x, t)$ is the laser electric field - which only lies in the transverse plane - and $\boldsymbol{V}_{\perp}=\boldsymbol{V}_{\perp}(x, t) \equiv\left[1 / n_{\mathrm{e}}(x, t)\right] \int \mathrm{d}^{3} v \boldsymbol{v}_{\perp} f_{\mathrm{e}}(x, \boldsymbol{v} ; t)$ is the projection of electron velocity moment onto the transverse plane. 

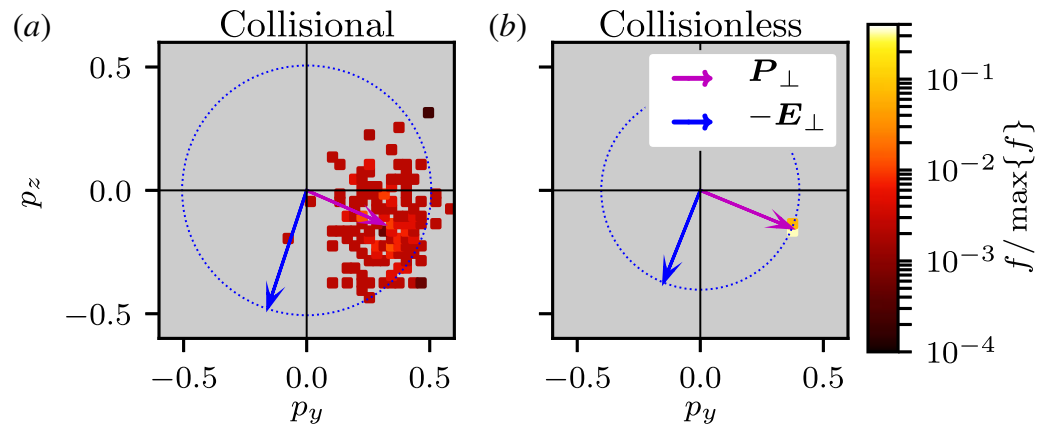

FIGURE 5. Electron transverse momentum distribution at $x=7.0-7.2 \mathrm{~nm}$ behind the ion front in the simplified simulations with fixed ions. The left and right panels correspond to collisional and collisionless simulations, respectively. The distributions are here recorded at $t=190 \mathrm{fs}$, which is well after the quasi-steady state has been reached, where $\boldsymbol{E}_{\perp}$ and $\boldsymbol{P}_{\perp}$ rotates (clockwise) in the transverse plane.

In a 1-D model, disregarding collisional effects, the transverse canonical momentum $\tilde{\boldsymbol{P}}_{\perp}=\boldsymbol{P}_{\perp}-\mathrm{e} \boldsymbol{A}_{\perp}$ is conserved, and $\tilde{\boldsymbol{P}}_{\perp}=0$. Hence $\boldsymbol{P}_{\perp}=\mathrm{e} \boldsymbol{A}_{\perp}$, where $\boldsymbol{A}_{\perp}$ and $\boldsymbol{P}_{\perp}$ are the transverse component of the magnetic vector potential and the electron momentum moment, defined analogously to $\boldsymbol{V}_{\perp}$. In quasi-steady state, $\boldsymbol{A}_{\perp}$ is just rotating in the transverse plane, so the electric field is $\boldsymbol{E}_{\perp} \equiv-\partial \boldsymbol{A}_{\perp} / \partial t=\omega A_{\perp}[\cos (\omega t) \hat{\boldsymbol{y}}-\sin (\omega t) \hat{\boldsymbol{z}}] / \sqrt{2}$, where $A_{\perp}=A_{\perp}(x)$ is the magnitude of the vector potential (necessarily transverse in one dimension). Importantly, the electric field vector is perpendicular to the vector potential and the magnitude of the electric field is $E_{\perp}=\omega A_{\perp}$. We therefore expect $\boldsymbol{P}_{\perp}$ and $\boldsymbol{E}_{\perp}$ to be perpendicular and their magnitudes - in normalized units - to be equal, $P_{\perp}=E_{\perp}$.

Figure 5 shows slices of the collisional $(a)$ and collisionless $(b)$ electron distributions in the transverse momentum plane at $t=190 \mathrm{fs}$, well after quasi-steady state has been reached, and in a thin slice 7.0-7.2 $\mathrm{nm}$ (one cell length) behind the immobile ion front edge of the plasma. If we were to evolve this picture in time, we would see the (negative) electric field $-\boldsymbol{E}_{\perp}$ rotate clockwise, along the marked-out circular path in figure 5; the mean momentum $\boldsymbol{P}_{\perp}$ would follow synchronously in this rotation. The most apparent difference between the collisional and collisionless distributions is the much larger momentum spread of the former, caused by collisional scattering of the electrons. In contrast to the collisionless case, $-\boldsymbol{E}_{\perp}$ and $\boldsymbol{P}_{\perp}$ are not equal in magnitude nor are they perfectly perpendicular. The missing transverse canonical momentum has been collisionally transferred to the ions, where it disappears from the simulation due to the ions being static. Note that if $-\boldsymbol{E}_{\perp}$ and $\boldsymbol{P}_{\perp}$ are not perfectly perpendicular in (3.1), then the absorbed power density $S$ is non-vanishing. ${ }^{3}$ We can express (3.1) as

$$
S=-n e \boldsymbol{E}_{\perp} \cdot \boldsymbol{V}_{\perp}=n e E_{\perp} V_{\perp} \sin (\alpha),
$$

where the phase angle between $-\boldsymbol{E}_{\perp}$ and $\boldsymbol{V}_{\perp}$ is $\pi / 2-\alpha$.

Figure 6 displays configuration space profiles of $E_{\perp}, V_{\perp}$ and $S-$ in dimensionless units - as well as $\sin (\alpha)$; the curves are produced from a time average over 21 time frames spanning $20 \mathrm{fs}$. In the collisionless case, we have $P_{\perp}(x)=E_{\perp}(x)$ and the phase

\footnotetext{
${ }^{3}$ For simplicity, we are ignoring relativistic effects in this discussion, which would otherwise complicate the relationship between $\boldsymbol{P}_{\perp}$ and $\boldsymbol{V}_{\perp}$.
} 


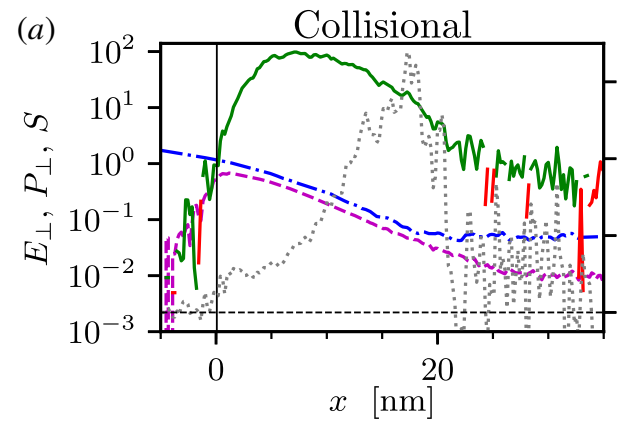

(b)

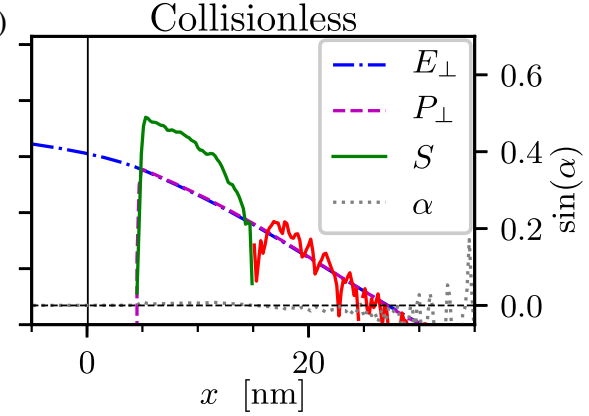

FIGURE 6. Magnitudes of the perpendicular electric field $E_{\perp}$ (blue dash-dotted) and mean electron transverse momentum $P_{\perp}$ (magenta dashed) as well as the absorbed power density $S$ (solid line, green and red for $S>0$ and $S<0$ respectively). Also shown is the phase shift $\sin (\alpha)$ (grey dotted) between $-\boldsymbol{E}_{\perp}$ and $\boldsymbol{V}_{\perp}$, where $\boldsymbol{V}_{\perp}$ is the mean electron transverse velocity moment of the distribution. The vertical black line marks the location of the transverse momentum planes plotted in figure 5. All values are expressed in dimensionless units.

shift angle $\alpha \simeq 0$ throughout the first $\simeq 25 \mathrm{~nm} \approx 8 l_{\mathrm{s}}$. Due to a finite spread in the electron transverse velocities, there will be a continuous exchange of electrons in the longitudinal direction not accounted for in the fluid description above, which induces a small deviation from $\alpha=0$ and hence $S \neq 0$. However, $S$ changes sign at $x \approx$ $15 \mathrm{~nm}$, beyond which the absorbed power is negative. In the collisional case, $P_{\perp}(x)$ is consistently smaller than $E_{\perp}(x)$. Furthermore, the phase shift $\sin (\alpha)$ is much larger, which is reflected in the about two orders of magnitude larger absorbed power $S$ than in the collisionless case.

A final note on the collisional case in figure $6(a)$ is the numerical artefact that causes both $E_{\perp}(x)$ and $P_{\perp}(x)$ to level off near $x=20 \mathrm{~nm}$. As the Monte Carlo collisional algorithm used in Smilei (Pérez et al. 2012) only conserves momentum statistically, a $P_{\perp}$ noise floor is generated which drives noise in $E_{\perp}$, i.e. the base level in figure $6(a)$. This effect could be alleviated by increasing the number of macro-particles. However, the absolute majority of the collisionally induced laser-energy absorption occurs in the region $x=5-15 \mathrm{~nm}$ and is therefore not significantly affected by the collisional noise floor.

\subsection{Parameter scans}

We have also performed parameter scans in order to investigate the dependencies of the collisional heating mechanism. One such scan has been in ionization, with either fixed ionization $\left(Z^{*}=11,19,24\right.$ and 27$)$ or self-consistent field and impact ionization. We have also conducted scans in laser intensity with $a_{0}$ ranging from 1 to 14 , and pulse durations from $t_{\mathrm{FWHM}}=15$ fs to $400 \mathrm{fs}$. The remaining parameters are as in $\S 2$.

Let us first consider the absorbed energy from the laser. Figure 7(a) shows the kinetic energy gain by the electrons and ions $(\Delta U)$ following the laser irradiation, for a scan in $a_{0}$ (colour coded) and a scan in pulse duration (shape coded). The value displayed on the horizontal axis is the laser pulse energy $J=I t_{\mathrm{FWHM}}[\pi / \log (4)]^{1 / 2} \propto$ $a_{0}^{2} t_{\mathrm{FWHM}}$, where $I$ is the laser intensity.

In the case of a constant pulse duration, $t_{\mathrm{FWHM}}=60 \mathrm{fs}$ (triangles), the trend scales like a power law with $\Delta U \propto J^{0.74}$ (dashed line) or $\Delta U \propto a_{0}{ }^{1.48}$, since $J \propto a_{0}{ }^{2}$. In 

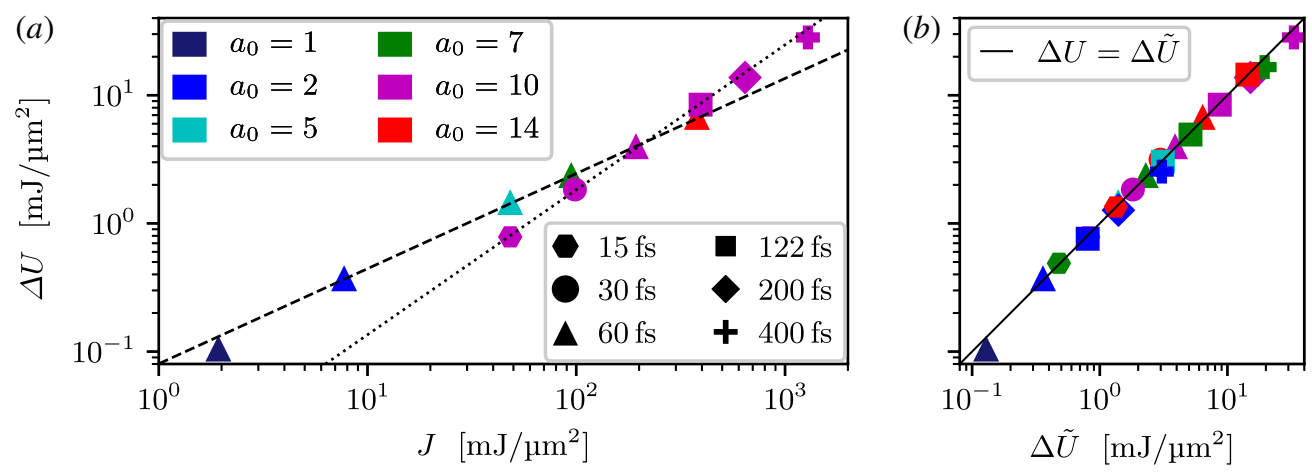

FIgURE 7. Total simulated kinetic energy gain $\Delta U$ against $(a)$ the laser energy $J$ and (b) the power law scaling $\Delta \tilde{U}$ for different combinations of laser parameter $a_{0}$ and duration $t_{\mathrm{FWHM}}$. Lines in panel $(a)$ indicate power law scalings: $\Delta U \propto J^{0.74} \propto a_{0}{ }^{1.48}$ at constant $t_{\mathrm{FWHM}}=60 \mathrm{fs}$ (dashed) and $\Delta U \propto J^{1.13} \propto t_{\mathrm{FWHM}}{ }^{1.13}$ at constant $a_{0}=10$ (dotted). These two power law scalings combine to give $\Delta \tilde{U}=0.23 \mathrm{~mJ} \mathrm{~mm}^{-2} \times a_{0}{ }^{1.48} \times$ $\left(t_{\mathrm{FWHM}} / 100 \mathrm{fs}\right)^{1.13}$, which is shown in panel $(b)$ to agree well with the full data set, also including parameter combinations not shown in $(a)$.

other words, the absorption efficiency scales as $\Delta U / J \propto J^{-0.26} \propto a_{0}{ }^{-0.52}$. The $a_{0}=1$ point seems to deviate from the above scaling, suggesting that it is mainly valid at relativistic intensities. Thus, $a_{0}=1$ was excluded from the fit. This scaling is similar to the $I^{-1 / 4}$ scaling of the normal skin effect, as described by Rozmus \& Tikhonchuk (1990). Comparing the collisional mean free path, $\lambda_{\mathrm{mfp}} \sim 20 \mathrm{~nm}$, to the skin depth, $l_{\mathrm{s}} \approx 6 \mathrm{~nm},{ }^{4}$ it is not completely clear that the normal skin effect $\left(\lambda_{\mathrm{mfp}} \lesssim l_{\mathrm{s}}\right)$ can be ruled out. However, our simulation results do not support some other scaling laws derived by Rozmus \& Tikhonchuk (1990) for normal skin effect, possibly due to the non-relativistic and simplified nature (constant intensity and semi-infinite plasma) of their analytic treatment.

The other scaling trend displayed in figure $7(a)$ is at constant $a_{0}=10$ (magenta). Here, the power law fit (dotted line) gives $\Delta U \propto J^{1.13} \propto t_{\mathrm{FWHM}}{ }^{1.13}$. In this case the absorption efficiency still has a weak positive scaling of $\Delta U / J \propto t_{\mathrm{FWHM}}{ }^{0.13}$. From the pulse duration scaling, we note that the $200 \mathrm{fs}$ and $400 \mathrm{fs}$ pulses begin to fall off below the scaling followed by the other data points, and they were thus also excluded from the fit. The decreasing trend with $t_{\text {FWHM }}$ in the long-pulse limit may be a consequence of the recirculation of hot electrons, which could lower the effective plasma collisionality in the irradiated region.

Combining the two above scalings yields the approximate scaling

$$
\Delta \tilde{U}=0.23 \mathrm{~mJ} \mu \mathrm{m}^{-2} \times a_{0}{ }^{1.48}\left(\frac{t_{\mathrm{FWHM}}}{100 \mathrm{fs}}\right)^{1.13}
$$

in the $\left(a_{0}, t_{\mathrm{FWHM}}\right)$ plane. Figure $7(b)$ shows this scaling to agree well with the full set of data, including data points where both $a_{0}$ and $t_{\mathrm{FWHM}}$ are varied. The observed scaling does break down at the low- $a_{0}$ or long-duration limits. However, the range of validity stretches over two orders of magnitude in pulse energy and a similar range in absorbed energy, and the laser parameters captured by this power law are

${ }^{4}$ The skin depth adjusted for collisions has been inferred from figure $6(a)$. 


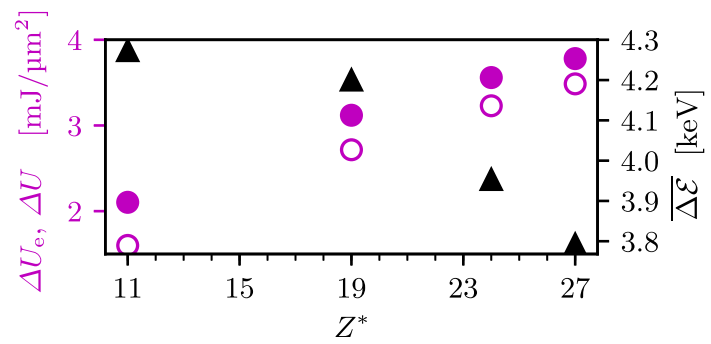

FIGURE 8. Collisional simulation with various (fixed) ionization degree $Z^{*}$. Left axis (magenta circles): gained kinetic energy by electrons and ions $\Delta U$ (filled circles) as well as only by electrons $\Delta U_{\mathrm{e}}$ (open circles). Right axis (black triangles): average kinetic energy gained by one electron $\overline{\Delta \mathcal{E}}$.

experimentally feasible and relevant to isochoric heating experiments. Collisional effects in general decrease at higher particle energies and the absorption happens through collisional scattering of the laser-driven electrons in the skin layer. Therefore, if the laser field $\left(a_{0}\right)$ is increased, so that the electrons in the skin layer reach higher energies, then the efficiency of the collisional absorption should decrease.

We also report on a scan in (fixed) ionization degree $Z^{*}$. Although this parameter cannot be controlled independently in experiments, this scan aims to provide insight into the target collisionality, which scales as $\left(Z^{*}\right)^{2}-$ ignoring other effects, e.g. individual particle energy. However, by varying $Z^{*}$ while keeping the ion density $n_{C u, 0}$ fixed, we inevitably also change the electron density $n_{\mathrm{e}, 0}=Z^{*} n_{C u, 0}$, which may introduce other density-related effects. Nevertheless, the electron density stays highly overcritical - the lowest electron density in this scan is $n_{\mathrm{e}, 0}=532.4 n_{\mathrm{c}}$ for $Z^{*}=11$. Figure 8 displays the energy absorbed by both electrons and ions $\Delta U$ (filled circles, left axis) and only by electrons $\Delta U_{\mathrm{e}}$ (open circles, left axis) for the different ionization degrees. The absorbed energy increases with $Z^{*}$, while a smaller fraction of the absorbed energy goes into the ions at higher $Z^{*}$.

Due to the accompanying changes in electron density, the average absorbed energy per electron $\overline{\Delta \mathcal{E}}$, also shown in figure 8 (black triangles, right axis), happens to decrease by approximately $15 \%$ from $Z^{*}=11$ to 27 . The decrease in $\overline{\Delta \mathcal{E}}$ with $Z^{*}$ may seem surprising if the dominant heating mechanism is collisional. However, this might be due to other density effects, such as the increased skin depth at lower electron density which allows a deeper laser penetration and thus a stronger laser-to-electron coupling efficiency. When we examine the electron energy spectra (not shown) in this scan, the Maxwellian-fitted bulk electron temperatures are all $T_{\mathrm{e}}=3.5 \pm 0.1 \mathrm{keV}$.

Besides just the pure amount of energy absorbed from the laser, we are also interested in how well thermalized the plasma is. As a measure of that, figure 9 shows the fraction of electron kinetic energy in the non-thermal electrons. This is calculated by the fraction of the energy in the high-energy tail to the total electron kinetic energy, $\int_{2 T_{\mathrm{e}}}^{\infty}\left[f_{\mathcal{E}}(\mathcal{E})-f_{\mathcal{E}}^{\mathrm{MJ}}(\mathcal{E})\right] \mathcal{E} \mathrm{d} \mathcal{E}$, where $f_{\mathcal{E}}^{\mathrm{MJ}}$ is a Maxwell-Jüttner distribution fitted to the bulk of the electron energy spectrum $f_{\mathcal{E}}(\mathcal{E})$ (as shown in figure 1) and $T_{\mathrm{e}}$ is the temperature inferred from the fit. Figure 9 shows scans in fixed ionization degree $Z^{*}(a)$, laser amplitude $a_{0}(b)$ and pulse duration $t_{\mathrm{FWHM}}(c)$. The displayed values are taken $200 \mathrm{fs}$ after the end of the laser pulse. Due to a varying heat transport speed, the fraction of non-thermal electron energy is only taken in the 

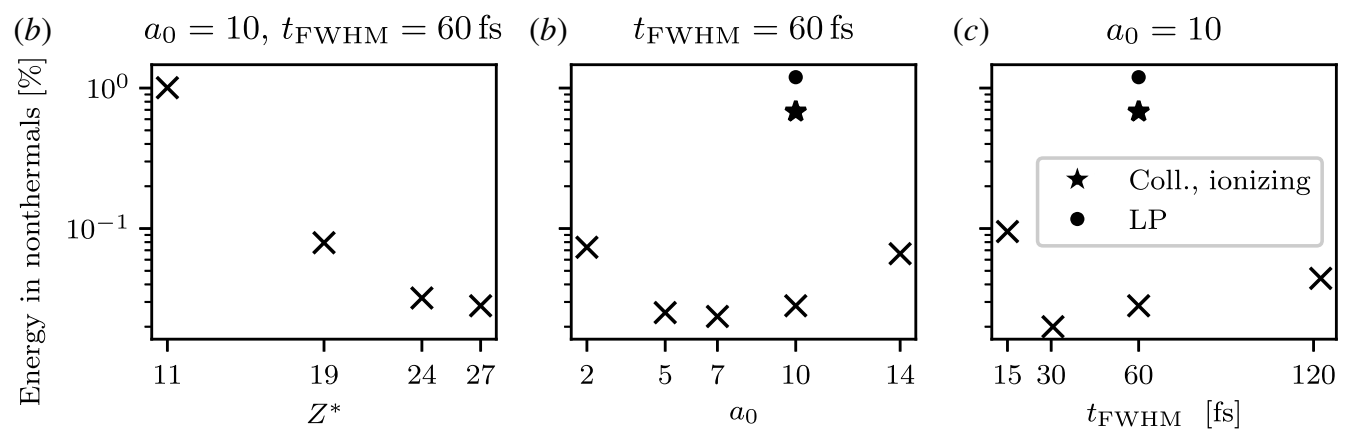

FIGURE 9. Fraction of the electron energy in non-thermal electrons, for collisional simulations, $200 \mathrm{fs}$ after the end of the laser pulse, for scans in $Z^{*}(a), a_{0}(b)$ and $t_{\text {FWHM }}(c)$ with $\mathrm{CP}$, marked by crosses. The value marked with a dot is from LP, and self-consistent ionization is shown as a star.

region in which the bulk electron temperature is no longer increasing. This should still give a representative estimate of the non-thermal fraction, since the fast electrons have already recirculated by the chosen time, see the $t=250 \mathrm{fs}$ panels of figure 3 . The precise values in figure 9 are sensitive to the choice of time and region to include, thus these results are only qualitative. Nevertheless, the general trends shown here are still representative of the observed situation - importantly, the relation between $\mathrm{LP}$ and $\mathrm{CP}$ is robust.

Figure $9(a)$ shows that there is a trend toward lower non-thermal fraction at higher ionization levels, which is consistent with the faster thermalization expected at high $Z^{*}$. This trend also suggests that the higher absorbed energy per electron at lower $Z^{*}$ (figure 8 ) is linked to a relative increase in the non-thermal population.

Regarding the scans in the laser amplitude and duration in figures $9(b)$ and $9(c)$, respectively, no clear trend appears to emerge among the CP laser pulses (marked by $x$ ). Then there are the self-consistent ionization (star) and LP (downward triangle) simulations: both have approximately one order of magnitude higher fraction of energy in non-thermal electrons than the equivalent (fixed-ionization, CP) counterpart. The higher fraction of non-thermal energy with LP stems from the $j \times B$ and vacuum heating mechanism. The higher non-thermal energy fraction with a self-consistent ionization process is discussed in conjunction with its phase-space distribution in figure 3 .

Even a very small fraction of non-thermals may affect the interpretation of X-ray diagnostics (Rosmej 1997; Chen et al. 2009; Renner \& Rosmej 2019), meaning that LP can be more intrusive than CP in WDM/HDM studies. We have also conducted simulations with a larger pre-expanded plasma (exponentially decaying density profile with a scale length of $80 \mathrm{~nm}$ ). While not presented here, those simulations show that LP can result in up to $\sim 10 \%$ of the electron kinetic energy in non-thermal electrons, which would of course be even more intrusive and significantly affect the $\mathrm{X}$-ray diagnostics. With $\mathrm{CP}$, the pre-plasma weakens the energy absorption by about a factor of two, but the fraction of energy in fast electrons stays $\lesssim 1 \%$.

\subsection{Two-dimensional simulation results}

Up to this point, all the results presented have been produced in 1-D simulations. However, to investigate the applicability of these results in higher dimensions, where 

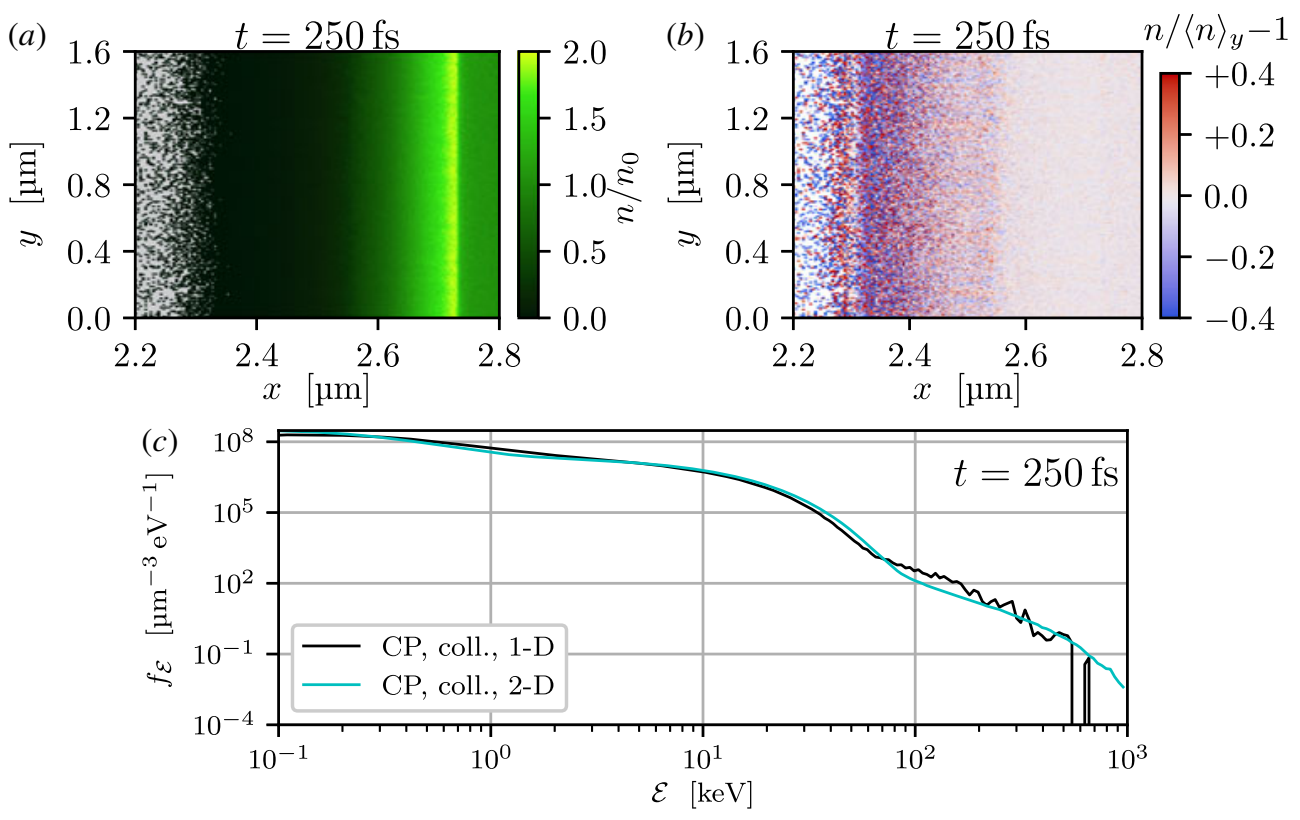

FIGURE 10. Map of the electron density $(a)$ in units of background density $n_{0}$ and the relative transverse variation of the electron density $(b)$ near the front target surface at 250 fs. Panel (c) shows a comparison of the electron energy spectra from the 1-D and 2-D simulations at the same time. The spectra are taken from the full simulation box. The transverse band of higher density at $x \approx 2.72 \mu \mathrm{m}$ in the panel $(a)$ corresponds to a shock front launched by the laser impact.

transverse plasma modulations can arise at the target boundary and affect the bulk heating and hot-electron generation (Kemp \& Divol 2016), we have performed a 2-D simulation at our baseline laser parameters (see $\S 2$ for details). A density map of the electron density near the illuminated target surface is shown in figure $10(a)$. The density is shown at $t=250$ fs. There is a transverse band of higher density at $x \approx$ $2.72 \mu \mathrm{m}$ that represents a shock front propagating into the plasma. Notably, this shock front remains straight, with no evidence of substantial density modulations.

To more clearly demonstrate the absence of transverse instability effects, the relative transverse variation of the electron density $n /\langle n\rangle_{y}-1$ is displayed in figure $10(b)$, where $\langle\cdot\rangle_{y}$ denotes a transverse average over the full width of the simulation box in $y$. Any transverse density structure should therefore be clearly visible in this representation. The substantial deviations from the average density observed in the low-density $\left(n_{e} \lesssim 0.2 n_{0}\right)$ region correspond to statistical noise due to a low number of computational particles in said region. Importantly, the deviations seen in figure $10(b)$ have no structure to them, and the same applies for the shock, suggesting that transverse effects are inoperative in the present highly collisional case (at least within the simulated time window).

Lastly, to confirm that the collisional heating behaves similarly in the 2-D and 1-D simulations, figure $10(c)$ shows the electron kinetic energy spectra of the corresponding 1-D and 2-D simulations at $t=250 \mathrm{fs}^{5}$ The spectra of the 2-D

\footnotetext{
${ }^{5}$ Due to the 2-D simulation having a smaller longitudinal box size, and thus the target front being located at $x=2.5 \mu \mathrm{m}$ instead of $x=7.5 \mu \mathrm{m}$, the times of comparison for the 1-D simulation are shifted by $15 \mathrm{fs}$
} 
and 1-D simulations are essentially the same. Although not shown here, the two spectra agree similarly well also at earlier times. Since the following thermalization process is almost entirely collisional, and thus independent of dimensionality, it is safe to conclude that the energy absorption is not affected by going from one to two dimensions, under the interaction conditions considered.

\section{Conclusions}

We have performed collisional and collisionless 1-D and 2-D PIC simulations and shown that a collisional, inverse bremsstrahlung, absorption can be used for strong plasma heating in a solid-density, high- $Z^{*}$ material, such as copper, with ultrahigh intensity, short-pulse lasers. Using $\mathrm{CP}$, the electron population quickly thermalizes to well-formed Maxwellian distributions suitable for experimental verification of HED physics models. The collisional simulations show that the target electrons are quickly heated to $T_{\mathrm{e}} \sim 3.5 \mathrm{keV}$ bulk temperature on a $\sim 300$ fs time scale. The target energy density reaches $\sim 10 \mathrm{Gbar}$, which is within the realm of ultrahigh energy density. The use of $\mathrm{CP}$ provides faster collisional thermalization of the electron population compared to LP, something which is valuable for experimental tests of HED atomic physics models. A test of the collisional absorption using a 2-D simulation, demonstrates the transferability of the 1-D results to higher dimensions. In contrast to previous work conducted with lower- $Z^{*}$ targets (Kemp \& Divol 2016), the high collisionality is not favourable for driving transverse plasma modulations, resulting in the same absorption levels in two dimensions as in one dimension, at least for CP.

We have carried out scans over laser parameters and ionization. The scans over laser settings show that the mechanism is robust to changes in the laser, over two orders of magnitude in laser energy, with lower intensity and longer pulses at the same laser pulse energy yielding better energy absorption. Also, the higher collisionality incurred from a higher ionization level improves energy absorption and electron thermalization. A more realistic simulation run with self-consistent ionization, including both impact and field ionization, reached $T_{\mathrm{e}} \sim 2.5 \mathrm{keV}$, confirming that collisional heating is still operational in a self-consistently ionized plasma, although its thermalization is then less complete than at fixed ionization, due to high-energy electrons generated through ionization events in strong-field regions.

\section{Acknowledgements}

The authors are grateful for fruitful discussions with L. Hesslow and T. Fülöp, as well as to M. Grech and F. Pérez for support with Smilei. This project has received funding from the European Research Council (ERC) under the European Union's Horizon 2020 research and innovation programme under grant agreement no. 647121, the Swedish Research Council (grant no. 2016-05012), and the Knut och Alice Wallenberg Foundation. The simulations were performed on resources provided by the Swedish National Infrastructure for Computing (SNIC) at Chalmers Centre for Computational Science and Engineering $\left(\mathrm{C}^{3} \mathrm{SE}\right)$ and High Performance Computing Center North $\left(\mathrm{HPC}^{2} \mathrm{~N}\right)$.

\section{REFERENCES}

later compared to the 2-D simulation, due to increased travel time for the laser pulse. The simulation time at which the 1-D spectrum is plotted is thus $t=265 \mathrm{fs}$. 
Bailey, J. E., Rochau, G. A., Iglesias, C. A., Abdallah, J., Macfarlane, J. J., Golovkin, I., Wang, P., Mancini, R. C., LaKe, P. W. \& Moore, T. C. 2007 Iron-plasma transmission measurements at temperatures above $150 \mathrm{eV}$. Phys. Rev. Lett. 99, 265002.

Bargsten, C., Hollinger, R., Capeluto, M. G., Kaymak, V., Pukhov, A., Wang, S., Rockwood, A., Wang, Y., Keiss, D., Tommasini, R. et al. 2017 Energy penetration into arrays of aligned nanowires irradiated with relativistic intensities: scaling to terabar pressures. Sci. Adv. 3, e1601558.

BAuer, D. \& Mulser, P. 2007 Vacuum heating versus skin layer absorption of intense femtosecond laser pulses. Phys. Plasmas 14 (2), 023301.

Bell, A. R. \& Kingham, R. J. 2003 Resistive collimation of electron beams in laser-produced plasmas. Phys. Rev. Lett. 91, 035003.

Brown, C. R. D., Hoarty, D. J., James, S. F., Swatton, D., Hughes, S. J., Morton, J. W., Guymer, T. M., Hill, M. P., Chapman, D. A., Andrew, J. E. et al. 2011 Measurements of electron transport in foils irradiated with a picosecond time scale laser pulse. Phys. Rev. Lett. 106, 185003.

Chen, S. N., Gregori, G., Patel, P. K., Chung, H.-K., Evans, R. G., Freeman, R. R., Garcia Saiz, E., Glenzer, S. H., Hansen, S. B. \& Khattak, F. Y. 2007 Creation of hot dense matter in short-pulse laser-plasma interaction with tamped titanium foils. Phys. Plasmas 14 (10), 102701.

Chen, S. N., Patel, P. K., Chung, H. K., Kemp, A. J., Le Pape, S., Maddox, B. R., Wilks, S. C., STEPhens, R. B.\& BEG, F. N. 2009 X-ray spectroscopy of buried layer foils irradiated at laser intensities in excess of $10^{20} \mathrm{~W} \mathrm{~cm}^{-2}$. Phys. Plasmas 16 (6), 062701.

Derouillat, J., Beck, A., Pérez, F., Vinci, T., Chiaramello, M., Grassi, A., Flé, M., Bouchard, G., Plotnikov, I., Aunai, N. et al. 2018 Smilei: a collaborative, open-source, multi-purpose particle-in-cell code for plasma simulation. Comput. Phys. Commun. 222, 351.

Dervieux, V., Loupias, B., Baton, S., Lecherbourg, L., Glize, K., Rousseaux, C., Reverdin, C., Gremillet, L., Blancard, C., Silvert, V. et al. 2015 Characterization of near-LTE, high-temperature and high-density aluminum plasmas produced by ultra-high intensity lasers. High Energy Density Phys. 16, 12-17.

DraKe, R. P. 2018 A journey through high-energy-density physics. Nucl. Fusion 59 (3), 035001.

Dyer, G. M., Bernstein, A. C., Cho, B. I., Osterholz, J., Grigsby, W., Dalton, A., Shepherd, R., Ping, Y., Chen, H., Widmann, K. et al. 2008 Equation-of-state measurement of dense plasmas heated with fast protons. Phys. Rev. Lett. 101, 015002.

Evans, R. G., Clark, E. L., Eagleton, R. T., Dunne, A. M., Edwards, R. D., Garbett, W. J., Goldsack, T. J., James, S., Smith, C. C., Thomas, B. R. et al. 2005 Rapid heating of solid density material by a petawatt laser. Appl. Phys. Lett. 86 (19), 191505.

FAussurier, G. \& BlANCARD, C. 2019 Pressure in warm and hot dense matter using the averageatom model. Phys. Rev. E 99, 053201.

Fujioka, S., Takabe, H., Yamamoto, N., Salzmann, D., Wang, F., Nishimura, H., Li, Y., Dong, Q., WANG, S., ZhANG, Y. et al. 2009 X-ray astronomy in the laboratory with a miniature compact object produced by laser-driven implosion. Nat. Phys. 8, 821.

Gregori, G., Hansen, S. B., Clarke, R., Heathcote, R., Key, M. H., King, J., Klein, R. I., IZUMi, N., MACKinNon, A. J., Moon, S. J. et al. 2005 Experimental characterization of a strongly coupled solid density plasma generated in a short-pulse laser target interaction. Contrib. Plasma Phys. 45 (3-4), 284-292.

GUILloRY, J. \& BENFord, G. 1972 Estimates of dense plasma heating by stable intense electron beams. Plasma Phys. 14 (12), 1131-1138.

Hoarty, D., Allan, P., James, S., Brown, C., Hobbs, L., Hill, M., Harris, J., Morton, J., Brookes, M., SHEPherd, R. et al. 2013a The first data from the Orion laser; measurements of the spectrum of hot, dense aluminium. High Energy Density Phys. 9 (4), 661-671.

Hoarty, D. J., Allan, P., James, S. F., Brown, C. R. D., Hobbs, L. M. R., Hill, M. P., Harris, J. W. O., Morton, J., Brookes, M. G., Shepherd, R. et al. 2013b Observations of the effect of ionization-potential depression in hot dense plasma. Phys. Rev. Lett. 110, 265003. 
HUbA, J. D. 2016 NRL Plasma Formulary. Naval Research Laboratory.

Joshipura, K., Vaishnav, B. \& LimbachiYA, C. 2006 Ionization and excitation of some atomic targets and metal oxides by electron impact. Pramana 66 (2), 403-414.

Kawahito, D. \& Kishimoto, Y. 2017 Multi-phase ionization dynamics of carbon thin film irradiated by high power short pulse laser. Phys. Plasmas 24 (10), 103105.

KemP, A. J. \& Divol, L. 2016 What is the surface temperature of a solid irradiated by a petawatt laser? Phys. Plasmas 23, 090703.

Knudson, M., Desjarlais, M. \& Dolan, D. 2008 Shock-wave exploration of the high-pressure phases of carbon. Science 322 (5909), 1822-1825.

Kramida, A., Ralchenko, Yu., Reader, J. \& NIST ASD Team 2018 NIST Atomic Spectra Database (ver. 5.6.1). ['Ionization Energies', 2019-08-15]. National Institute of Standards and Technology, Gaithersburg, MD. doi:10.18434/T4W30F.

Kruer, W. L. \& Estabrook, K. $1985 \mathrm{~J} \times$ B heating by very intense laser light. Phys. Fluids 28, 430-432.

Le Pape, S., Berzak Hopkins, L. F., Divol, L., Pak, A., Dewald, E. L., Bhandarkar, S., Bennedetti, L. R., Bunn, T., Biener, J., Crippen, J. et al. 2018 Fusion energy output greater than the kinetic energy of an imploding shell at the national ignition facility. Phys. Rev. Lett. 120, 245003.

Lovelace, R. V. \& SudAn, R. N. 1971 Plasma heating by high-current relativistic electron beams. Phys. Rev. Lett. 27, 1256-1259.

Mančić, A., Lévy, A., Harmand, M., Nakatsutsumi, M., Antici, P., Audebert, P., Combis, P., Fourmaux, S., Mazevet, S., Peyrusse, O. et al. 2010 Picosecond short-range disordering in isochorically heated aluminum at solid density. Phys. Rev. Lett. 104, 035002.

Martinolli, E., Koenig, M., Baton, S. D., Santos, J. J., Amiranoff, F., Batani, D., Perelli-Cippo, E., Scianitti, F., Gremillet, L., Mélizzi, R. et al. 2006 Fast-electron transport and heating of solid targets in high-intensity laser interactions measured by $\mathrm{K} \alpha$ fluorescence. Phys. Rev. E 73, 046402.

May, J., Tonge, J., Fiuza, F., Fonseca, R. A., Silva, L. O., Ren, C. \& Mori, W. B. 2011 Mechanism of generating fast electrons by an intense laser at a steep overdense interface. Phys. Rev. E 84, 025401.

More, R. M. 1983 Atomic processes in high-density plasma. In Atomic and Molecular Physics of Controlled Thermonuclear Fusion (ed. C. J. Joachain \& D. E. Post), pp. 399-439. Plenum Publishing Corporation.

NAnBU, K. 1997 Theory of cumulative small-angle collisions in plasmas. Phys. Rev. E 55, 4642-4652.

NAnbU, K. \& YonemuRA, S. 1998 Weighted particles in Coulomb collision simulations based on the theory of a cumulative scattering angle. J. Comput. Phys. 145 (2), 639-654.

Nettelmann, N., Holst, B., Kietzmann, A., French, M., Redmer, R. \& Blaschke, D. 2008 $A b$ initio equation of state data for hydrogen, helium, and water and the internal structure of Jupiter. Astrophys. J. 683 (2), 1217-1228.

Nilson, P. M., Solodov, A. A., Myatt, J. F., Theobald, W., JaAnimagi, P. A., Gao, L., Stoeckl, C., Craxton, R. S., Delettrez, J. A., YaAkobi, B. et al. 2010 Scaling hot-electron generation to high-power, kilojoule-class laser-solid interactions. Phys. Rev. Lett. 105, 235001.

Nilson, P. M., Theobald, W., Myatt, J. F., Stoeckl, C., Storm, M., Zuegel, J. D., Betti, R., Meyerhofer, D. D. \& SAngster, T. C. 2009 Bulk heating of solid-density plasmas during high-intensity-laser plasma interactions. Phys. Rev. E 79, 016406.

Patel, P. K., Mackinnon, A. J., Key, M. H., Cowan, T. E., Foord, M. E., Allen, M., Price, D. F., Ruhl, H., Springer, P. T. \& Stephens, R. 2003 Isochoric heating of solid-density matter with an ultrafast proton beam. Phys. Rev. Lett. 91, 125004.

Pérez, F., Gremillet, L., Decoster, A., Drouin, M. \& Lefebvre, E. 2012 Improved modeling of relativistic collisions and collisional ionization in particle-in-cell codes. Phys. Plasmas 19, 083104. 
Pérez, F., Gremillet, L., Koenig, M., Baton, S. D., Audebert, P., Chahid, M., Rousseaux, C., Drouin, M., Lefebvre, E., Vinci, T. et al. 2010 Enhanced isochoric heating from fast electrons produced by high-contrast, relativistic-intensity laser pulses. Phys. Rev. Lett. 104, 085001.

Purvis, M. A., Shlyaptsev, V. N., Hollinger, R., Bargsten, C., Pukhov, A., Prieto, A., WANG, Y., LUther, B. M., YIN, L., WANG, S. et al. 2013 Relativistic plasma nanophotonics for ultrahigh energy density physics. Nat. Photon. 7, 796-800.

Remington, B. A. 2005 High energy density laboratory astrophysics. Plasma Phys. Control. Fusion 47, A191-A203.

Renaudin, P., Blancard, C., Clérouin, J., Faussurier, G., Noiret, P. \& Recoules, V. 2003 Aluminum equation-of-state data in the warm dense matter regime. Phys. Rev. Lett. 91, 075002 .

Renner, O. \& Rosmej, F. B. 2019 Challenges of x-ray spectroscopy in investigations of matter under extreme conditions. Matter Radiat. Extr. 4 (2), 024201.

Robinson, A., Strozzi, D., Davies, J., Gremillet, L., Honrubia, J., Johzaki, T., Kingham, R., Sherlock, M. \& Solodov, A. 2014 Theory of fast electron transport for fast ignition. Nucl. Fusion 54 (5), 054003.

RosmeJ, F. B. 1997 Hot electron x-ray diagnostics. J. Phys. B 30 (22), L819-L828.

Ross, M. 1981 The ice layer in Uranus and Neptune - diamonds in the sky? Nature 292 (5822), 435-436.

Rozmus, W. \& Tikhonchuk, V. T. 1990 Skin effect and interaction of short laser pulses with dense plasmas. Phys. Rev. A 42, 7401.

Santos, J. J., Vauzour, B., Touati, M., Gremillet, L., Feugeas, J.-L., Ceccotti, T., Bouillaud, R., Deneuville, F., Floquet, V., Fourment, C. et al. 2017 Isochoric heating and strong blast wave formation driven by fast electrons in solid-density targets. New J. Phys. 19 (10), 103005.

Sawada, H., Sentoku, Y., Yabuuchi, T., Zastrau, U., Förster, E., Beg, F. N., Chen, H., Kemp, A. J., Mclean, H. S., Patel, P. K. et al. 2019 Monochromatic 2D K $\alpha$ emission images revealing short-pulse laser isochoric heating mechanism. Phys. Rev. Lett. 122, 155002.

Sherlock, M., Hill, E. G., Evans, R. G., Rose, S. J. \& Rozmus, W. 2014 In-depth plasma-wave heating of dense plasma irradiated by short laser pulses. Phys. Rev. Lett. 113, 255001.

Siminos, E., Grech, M., Skupin, S., Schlegel, T. \& TikhonchuK, V. T. 2012 Effect of electron heating on self-induced transparency in relativistic-intensity laser-plasma interactions. Phys. Rev. E 86, 056404.

Siminos, E., Grech, M., Svedung Wettervik, B.\& FülöP, T. 2017 Kinetic and finite ion mass effects on the transition to relativistic self-induced transparency in laser-driven ion acceleration. New J. Phys. 19 (12), 123042.

Sundström, A., Siminos, E., Gremillet, L. \& Pusztai, I. 2020 Collisional effects on the ion dynamics in thin-foil targets driven by an ultraintense short pulse laser. Plasma Phys. Control. Fusion arXiv:2002.10935; (submitted). 